\title{
Current trends of the magnetoelectric effect
}

\author{
M. Fiebig ${ }^{1}$ and N.A. Spaldin ${ }^{2}$ \\ 1 HISKP, Universität Bonn, Nussallee 14-16, 53115 Bonn, Germany \\ 2 Materials Department, University of California, Santa Barbara, California 93106-5050, USA
}

Received 8 July 2009

Published online 13 October 2009 - (c) EDP Sciences, Società Italiana di Fisica, Springer-Verlag 2009

\begin{abstract}
This article is a summary report on the Topical Issue on Magnetoelectric Interaction Phenomena in Crystals. The topics discussed in this special issue are reviewed and discussed in the context of prior and current research activities in the field. New developments during the past five years are pointed out and an outlook on possible future trends in research on the magnetoelectric effect is given.
\end{abstract}

PACS. 70 Condensed matter: electronic structure, electrical, magnetic and optical properties - 68 Surfaces and interfaces; thin films and nanosystems (structure and nonelectronic properties)

\section{Introduction}

This Topical Issue is devoted to the magnetoelectric (ME) effect in strongly correlated condensed-matter systems. According to the original definition, which was motivated by Pièrre Curie as early as 1894, the ME effect denotes the induction of a magnetization $\boldsymbol{M}$ proportional to an electric field $\boldsymbol{E}$ and of an electric polarization $\boldsymbol{P}$ proportional to a magnetic field $\boldsymbol{H}$. This is expressed by $P_{i}=\alpha_{i j} H_{j}$ and $M_{i}=\mu_{0} \alpha_{j i} E_{j}$, where $\hat{\alpha}$ is the ME coupling tensor $[1,2]$. Over time, an increasingly general concept of ME behavior has been adopted. First of all, the linear ME effect described above has been extended to include higher-order ME effects described by terms such as $P_{i}=\gamma_{i j k} E_{j} H_{k}$. In addition to this obvious extension, it is currently common to denote any sort of interaction between the magnetic and electric properties of matter as a ME effect. As a result, the ME effect has become inextricably linked to the topic of multiferroics; that is materials exhibiting more than one form of (anti-) ferroic order in the same phase [2,3]. Among these, the magnetic ferroelectrics are relevant to our discussion of magnetoelectricity, and are often referred to as "ME multiferroics" [4]. In such multiferroics, the electric or magnetic fields can trigger magnetic or electric phase transitions instead of merely inducing a magnetization or polarization, resulting in so-called "giant ME effects". Developments such as ME phase control through giant $\mathrm{ME}$ effects and demonstrations of magnetic domain switching by electric fields (and vice versa) have led to an impressive resurgence of interest in ME coupling phenomena, as shown in Figure 1. This is no doubt driven in part by the technological appeal of controlling magnetism by electric fields, but at the same time ME effects in multiferroics and related materials offer fascinating new perspectives from the point of view of basic research.

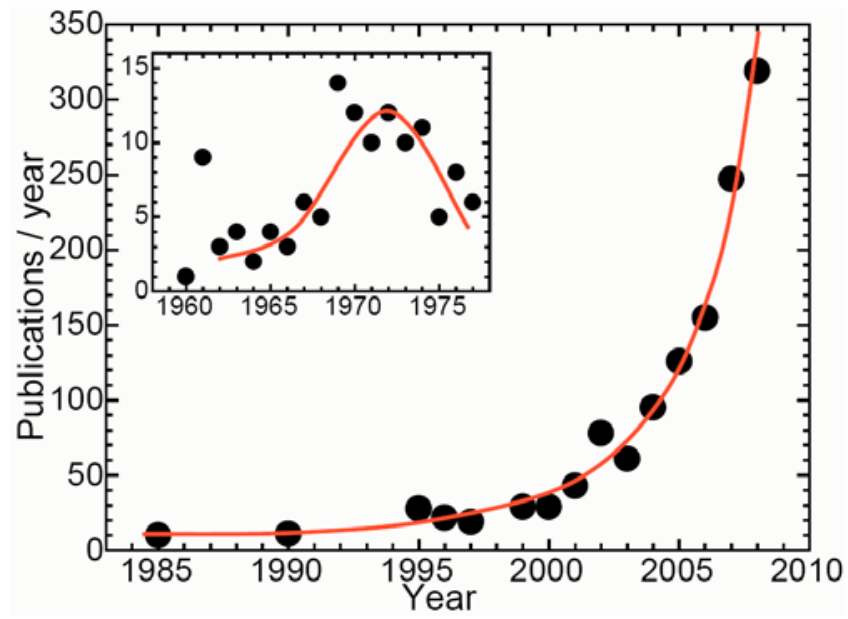

Fig. 1. Number of citations per year under the keyword "magnetoelectric" according to the ISI Web of Knowledge ${ }^{\mathrm{SM}}$. After a temporary boom in the early 1970s research on the ME effect languished until its recent and ongoing exponential upturn.

This evolving view of the ME effect over the last 40 years has been reflected in a series of international meetings entitled MEIPIC (Magnetoelectric Interaction Phenomena in Crystals). The first MEIPIC meeting [5] was conceived as an "East-meets-West" conference and was held in Seattle (USA) in 1972 in the midst of the Cold War; ever since MEIPIC has endeavored to retain this bilateral aspect. MEIPIC-2 to MEIPIC-5 were held in Ascona (Switzerland, 1993) [6], Novgorod (Russia, 1996) [7], Novgorod (Russia, 2001) [8], and Sudak (Ukraine, 2003) [9]. With MEIPIC-6, held at UC Santa Barbara (USA) in January 2009 and sponsored by the National Science Foundation-supported International Center for Materials 
Research, the meeting finally returned to its country of origin.

It is the purpose of this Topical Issue to emphasize the relationship between the ME effect as it is discussed today in the context of multiferroics, and the ME effect as it was perceived prior to the year 2000 and at the various MEIPIC meetings. In building this bridge, the editors of this Topical Issue found discussions with Igor Dzyaloshinskii and Hans Schmid, two pioneers of the ME effect in the East and the West, respectively, particularly instructive.

\section{Magnetically induced ferroelectricity}

The discussion of the relationship between multiferroic order and the ME effect is currently focussed on the class of multiferroics known as "magnetically induced ferroelectrics", which are a type of multiferroic with particularly strong ME coupling [10]. In these materials, which were first identified in the 1970s during the flurry of activity motivated by MEIPIC-1 [11], a non-centrosymmetric magnetic ordering breaks the inversion symmetry and induces a ferroelectric state. The polarization is therefore intimately coupled to the magnetic ordering. For example the magnetic and electric transition temperatures into the multiferroic state are identical, and reorientation or suppression of one order parameter using its conjugate field directly affects the other. Multiferroics of this type may be termed "joint-order-parameter multiferroics" in contrast to "split-order multiferroics", in which magnetic and ferroelectric order emerge independently [12] (here $\mathrm{BiFeO}_{3}$ is the prototype [13]). Representatives of magneticallyinduced ferroelectrics that are emphasized in this Topical Issue are orthorhombic $\mathrm{TbMnO}_{3}$ and $\mathrm{TbMn}_{2} \mathrm{O}_{5}$. Symmetric and antisymmetric exchange as the principal microscopic mechanisms driving magnetically-induced ferroelectricity [14-33] are discussed in many of the articles. In addition, the concept of spin currents (as opposed to ohmic charge currents) [17] is used to describe multiferroicity in $\mathrm{TbMnO}_{3}$ and related compounds.

\section{New geometries for composites}

As early as 1972 it was known that a ME response can be generated as a product effect in composite materials composed of a piezoelectric and a magnetostrictive constituent [34]. In such composites, an AC magnetic field deforms the magnetostrictive constituent and the deformation is transferred mechanically to the piezoelectric constituent, thus inducing an AC voltage [1]. A very pronounced $\mathrm{ME}$ response of up to $1 \mathrm{kV} /(\mathrm{cm} \cdot \mathrm{Oe})$ can be obtained under ambient conditions [35], albeit only at microwave frequencies. In this Topical Issue recent advances in construction and architecture of ME composites are presented [36,37]; these advances have enabled filters with electric-field-tuneable magnetic resonance frequencies and high quality factors $[38,39]$. In another development, a gyrator, which is a device converting between active and reactive impedances and electrical currents and voltages, based on composite multiferroics is introduced [40].

In addition to such strain-mediated composites, epitaxial heterostructures with a variety of novel ME coupling effects based on subtle interface functionalities are discussed. It has recently been shown that strong ME coupling can be obtained in heterostructures of antiferromagnetic ferroelectric $\mathrm{BiFeO}_{3}$ that is exchange-biased to a ferromagnetic metal [41]. Here an electric voltage modifies the antiferromagnetic state in the multiferroic, which in turn modifies the ferromagnetic metal through exchange-bias coupling. Much discussion centers around understanding the complex ferroelectric domain structure of $\mathrm{BiFeO}_{3}$ and its coupling to the magnetic domain state $[42,43]$. In addition it is now known that the properties of oxides with strong electronic correlations can be elegantly controlled by growing them on piezoelectric substrates [44-46]. Electric voltages determine the lattice constant of the substrate and are used to strain the oxide film grown onto it in a controlled, reversibly tunable way, thus changing its magnetic and electric properties.

\section{Ferrotoroidic order}

A lively discussions in the present literature is devoted to the claim of a fourth form of ferroic order, termed "ferrotoroidicity". In contrast to the established forms of ferroic order - ferromagnetism, ferroelectricity, and ferroelasticity - the ferrotoroidic state is characterized by a spontaneous uniform alignment of magnetic vortices $[3,47,48]$. The ferrotoroidic state violates both the spatial and the temporal inversion symmetry. Since this is exactly the requirement for linear ME coupling, ferrotoroidics form a class of materials inherently allowing the linear ME effect. Indeed toroidal concepts have been recently used to design novel magnetoelectric materials [49]. In this Topical Issue a microscopic framework for defining ferrotoroidic systems is presented [50,51]. The experimental confirmation of ferrotoroidic order in the form of an observation of ferrotoroidic domains is reported $[52,53]$. Importantly, a symmetry concept that includes the established forms of ferroic and antiferroic order as well as ferrotoroidicity, and allows extensions to additional forms of spontaneous long-range order is presented $[54,55]$.

\section{Symmetry issues}

When Curie predicted the linear ME effect in 1894 he already pointed out that symmetry aspects are an essential aspect of its occurrence. Only recently, macroscopic effects involving magnetic, electric, elastic, and toroidic interactions were distinguished based on the respective symmetry [3]. Discussion in this Topical Issue is focused on the relation between symmetry and multiferroics/ferrotoroidics as an extension of the concepts of spontaneous long-range order. A systematic classification of the number of domains to be expected in ferroics, antiferroics, and multiferroics 
of any type is presented [54-56], as well as the possibility of extending these classification schemes to superconductance phenomena with their $\mathrm{U}(1)$ and $\mathrm{SU}(2)$ gauge symmetries.

In a specific example two compounds, $\mathrm{BiFeO}_{3}$ and $\mathrm{FeTiO}_{3}$ with $\mathrm{Fe}$ ions on different lattice sites, are compared in this Topical Issue regarding the relation between symmetry and macroscopic properties $[50,51,57]$. It is shown that the local symmetry of the Fe ion is crucial in determining the strength and nature of the ME coupling in the two compounds.

\section{Extended concepts of ME coupling}

As mentioned above, the classification of a system or process as ME is continuously being generalized. This is reflected in the diversity of novel contributions to this Topical Issue. At a very fundamental level the occurrence of a paramagnetic response from diamagnetic ferroelectrics is predicted [58]. In another fundamental contribution a relativistic concept for the linear ME effect is introduced $[59,60]$. Finally, in addition to the coupling between electric fields and magnetization, the coupling between electric fields and the gradient of magnetization, termed flexoelectric effect, is discussed [61,62]. Experimentally, the role of "electromagnons" - dielectric resonances in the magnetically ordered state that can be quenched by a static magnetic field - in extending the ME effect into the $\mathrm{THz}$ range is debated $[63,64]$. Separation of the electromagnon from two-magnon effects at $\mathrm{THz}$ frequencies is an important theoretical issue [65-67].

Evidence for local ME effects are found in the form of conducting domain walls in $\mathrm{BiFeO}_{3}$ [69], the only robust ambient multiferroic known at present. An extension from long-range to short-range ME correlations is introduced in the form of a ME multiglass [70-72]. In contrast to ferroics, which are characterized by uniform longrange order, glasses are dominated by frustration so that the alignment of electric dipoles or magnetic moments occurs only locally. Specifically, $\mathrm{Mn}^{2+}$-doped $\mathrm{SrTiO}_{3}$ and $\mathrm{K}_{0.97} \mathrm{Mn}_{0.03} \mathrm{TaO}_{3}$ have been shown to form a glassy state both in a magnetic and a dielectric sense so that the terminology "multiglass" suggests itself. While the linear ME effect is forbidden in a multiglass, a variety of higher-order ME effects are possible. Finally, an entirely new realm for ME effects in multiferroics is opened by the discovery of coexisting magnetic and electric phases in organic compounds. The ME behavior of the multiferroic state in organics can be quite peculiar, requiring magnetic field pulse sequences instead of magnetic fields for a modification of the ferroelectric state [68].

\section{Proposed future developments in magnetoelectrics research}

Because of the rapid progress in research on multiferroics and the ME effect at present a prediction of research trends beyond the year 2010 is difficult. However, several topics of emerging visibility are identified in this Topical Issue, in recent publications, and at current international meetings.

Present research activities are mostly devoted to oxide compounds and bulk samples or epitaxial films. Here it is expected that new types of materials will be included in the future. Non-oxide compounds such as spinels and organic multiferroics are promising classes of materials. As mentioned before, even superconductors may become involved. In addition structured systems are expected to play a greater role. This may involve correlations at interfaces such as two-dimensional interface states [73-75], field effects, or even ME metamaterials in which both the inversion symmetry and the time-reversal symmetry are structurally broken [76,77].

The search for new ways to combine and couple magnetism and ferroelectricity in single-phase multiferroics will almost certainly continue. Here novel mechanisms such as charge ordering, which has been proposed in manganites $[78,79]$, nickelates and even long-studied magnetite, are ripe for exploration.

Application of the term "magnetoelectric" will be extended even further to involve spin-charge correlations on a general basis. A stronger connection to the field of spintronics may be anticipated [80]. Aside from the microwave $\mathrm{ME}$ effect in composite multiferroics and the discussion of electromagnons, dynamic ME effects are still a largely neglected field of research. Here the discussion of ME behavior in the optical range such as in Ref. [81] may be expanded. In addition, temporal aspects of ME correlations may be investigated. How do the spin and charge sublattices relax after driving them off equilibrium, for example by irradiation of an intense light pulse? What are ME cross-correlations coupling the sublattices to one another? Finally, how does a voltage-induced magnetic switching process, such as in a joint-order-parameter multiferroic, evolve in a time-resolved experiment?

In conclusion, the editors are happy to present the collection of articles of this Topical Issue as an inspiring overview of the contemporary discussion on the ME effect. We hope that it will stimulate further important contributions and novel research trends in experimental exploration and theoretical concepts.

Lively discussions on the past and future of the ME effect with Hans Schmid and Igor Dzyaloshinskii are appreciated. M. F. thanks the SFB 608 of the DFG and the EU-STREP MaCo$\mathrm{MuFi}$ and N.A.S. thanks the National Science Foundation under grant number Award No. DMR-0605852 for supporting their respective research activities on the ME effect. The editors thank the IMI Program of the National Science Foundation under Award No. DMR04-09848 for facilitating their collaborations.

\section{References}

1. M. Fiebig, J. Phys. D 38, 123 (2005)

2. J.-P. Rivera, Eur. Phys. J. B, DOI : $10.1140 /$ epjb/e2009-00336-7 
3. H. Schmid, J. Phys.: Condens. Matter 20, 434201 (2008)

4. N.A. Spaldin, M. Fiebig, Science 309, 391 (2005)

5. Magnetoelectric Interaction Phenomena in Crystals, edited by A.J. Freeman, H. Schmid (Gordon and Breach, London, 1975), Proceedings of MEIPIC-1, held May, 21-24, 1973, in Seattle, USA

6. Ferroelectrics $\mathbf{1 6 1}+\mathbf{1 6 2}$ (1994), edited by H. Schmid, A. Janner, H. Grimmer, J.P. Rivera, Z.G. Ye; Proceedings of MEIPIC-2, held September 13-18, 1993, in Ascona, Switzerland

7. Ferroelectrics 204 (1997), edited by M. Bichurin; Proceedings of MEIPIC-3, held September 16-20, 1996 in Novgorod, Russia

8. Ferroelectrics $\mathbf{2 7 9}+\mathbf{2 8 0}$ (2002), edited by M. Bichurin; Proceedings of MEIPIC-4, held October 16-19, 2001 in Novgorod, Russia

9. Magnetoelectric Interaction Phenomena in Crystals, edited by M. Fiebig, V.V. Eremenko, I.E. Chupis (Kluwer, Dordrecht, 2004). Proceedings of MEIPIC-5, held September 21-24, 2003, in Sudak, Ukraine

10. T. Kimura, Annu. Rev. Mater. Res. 37, 387 (2007)

11. R.E. Newnham, J.J. Kramer, W.A. Schulze, L.E. Cross, J. Appl. Phys. 49, 6088 (1978)

12. D. Khomskii, Physics 2, 20 (2009)

13. J. Wang, J.B. Neaton, H. Zheng, V. Nagarajan, S.B. Ogale, B. Liu, D. Viehland, V. Vaithyanathan, D.G. Schlom, U.V. Waghmare, N.A. Spaldin, K.M. Rabe, M. Wuttig, R. Ramesh, Science 299, 1719 (2003)

14. I.A. Sergienko, E. Dagotto, Phys. Rev. B 73, 094434 (2006)

15. N. Aliouane, D.N. Argyriou, J. Strempfer, I. Zegkinoglou, S. Landsgesell, M.v. Zimmermann, Phys. Rev. B 73, 020102(R) (2006)

16. I.A. Sergienko, C. Şen, E. Dagotto, Phys. Rev. Lett. 97, $227204(2006)$

17. H. Katsura, N. Nagaosa, A.V. Balatsky, Phys. Rev. Lett. 95, $057205(2005)$

18. C. Jia, S. Onoda, N. Nagaosa, J.H. Han, Phys. Rev. B 74, $224444(2006)$

19. L.C. Chapon, P.G. Radaelli, G.R. Blake, S. Park, S.-W. Cheong, Phys. Rev. Lett. 96, 097601 (2006)

20. A.S. Moskvin S.-L. Drechsler, Phys. Rev. B 78, 024102 (2008)

21. K. Yamauchi, F. Freimuth, S. Blügel, S. Picozzi, Phys. Rev. B 78, 014403 (2008)

22. C. Wang, G.-C. Guo, L. He, Phys. Rev. B 77, 134113 (2008)

23. G. Giovannetti, J. van den Brink, Phys. Rev. Lett. 100, $227603(2008)$

24. S. Picozzi, K. Yamauchi, B. Sanyal, I.A. Sergienko, E. Dagotto, Phys. Rev. Lett. 99, 227201 (2007)

25. R. Valdés Aguilar, A. B. Sushkov, S. Park, S.-W. Cheong, H. D. Drew Phys. Rev. B 73, 184404 (2006)

26. L.C. Chapon, G.R. Blake, M.J. Gutmann, S. Park, N. Hur, P.G. Radaelli, S.-W. Cheong, Phys. Rev. Lett. 93, 177402 (2004)

27. V. Polyakov, V. Plakhty, M. Bonnet, P. Burlet, L.-P. Regnault, S. Gavrilov, I. Zobkalo, O. Smirnov, Physica B 297, 208 (2001)

28. I. Kagomiya, S. Matsumoto, K. Kohn, Y. Fukuda, T. Shoubu, H. Kimura, Y. Noda, N. Ikeda, Ferroelectrics 286, $167(2003)$

29. G.R. Blake, L.C. Chapon, P.G. Radaelli, S. Park, N. Hur, S.-W. Cheong, J. Rodríguez-Carvajal, Phys. Rev. B 71, $214402(2005)$
30. B. Mihailova, M.M. Gospodinov, B. Güttler, F. Yen, A.P. Litvinchuk, M.N. Iliev, Phys. Rev. B 73, 172301 (2006)

31. A.F. García-Flores, E. Granado, H. Martinho, R.R. Urbano, C. Rettori, E.I. Golovenchits, V.A. Sanina, S.B. Oseroff, S. Park, S.-W. Cheong, Phys. Rev. B 73, 104411 (2006)

32. A. Malashevich, D. Vanderbilt, Eur. Phys. J. B, DOI : 10.1140/epjb/e2009-00208-2

33. A.S. Moskvin, S.L. Drechsler, Eur. Phys. J. B, DOI : 10.1140/epjb/e2009-00264-6

34. J. van Suchtelen, Philips Res. Rep. 27, 28 (1972)

35. J. Zhai, S. Dong, Z. Xing, J. Li, D. Viehland, Appl. Phys. Lett. 89, 083507 (2006)

36. V.M. Petrov, V.V. Zibtsev, G. Srinivasan, Eur. Phys. J. B, DOI : 10.1140/epjb/e2009-00207-3

37. W.-M. Zhu, L.-W. Su, Z.-G. Ye, Eur. Phys. J. B, DOI : 10.1140/epjb/e2009-00252-x

38. G. Srinivasan, A.S. Tatarenko, V. Mathe, M.I. Bichurin Eur. Phys. J. B, DOI : 10.1140/epjb/e2009-00227-y

39. S. Shastry, G. Srinivasan, M.I. Bichurin, V. M. Petrov, A.S. Tatarenko, Phys. Rev. B 70, 064416 (2004)

40. J. Zhai, J. Gao, C. DeVreugd, J. Li, D. Viehland, A.V. Filippov, M.I. Bichurin, D.V. Drozdov, G.A. Semenov, S. Dong, Eur. Phys. J. B, DOI : 10.1140/epjb/e2009-00318-9

41. L.W. Martin, Y.-H. Chu, M.B. Holcomb, M. Huijben, P. Yu, S.-J. Han, D. Lee, S.X. Wang, R. Ramesh, Nano Lett. 8, 2050-2055 (2008)

42. T. Zhao, A. Scholl, F. Zavaliche, K. Lee, M. Barry, A. Doran, M.P. Cruz, Y.H. Chu, C. Ederer, N.A. Spaldin, R.R. Das, D.M. Kim, S.H. Baek, C.B. Eom, R. Ramesh, Nat. Mater. 5, 823 (2006)

43. D. Lebeugle, D. Colson, A. Forget, M. Viret, A.M. Bataille, A. Gukasov, Phys. Rev. Lett. 100, 227602 (2008)

44. C. Thiele, K. Dörr, O. Bilani, J. Rödel, L. Schultz, Phys. Rev. B 75, 054408 (2007)

45. A.D. Rata, A. Herklotz, K. Nenkov, L. Schultz, K. Dörr, Phys. Rev. Lett. 100, 076401 (2008)

46. K. Dörr, O. Bilani-Zeneli, A. Herklotz, A.D. Rata, K. Boldyreva, J.-W. Kim, M.C. Dekker, K. Nenkov, L. Schultz, M. Reibold, H. Lichte, Eur. Phys. J. B, DOI : 10.1140/epjb/e2009-00296-x

47. V.L. Ginzburg, A.A. Gorbatsevich, Y.V. Kopayev, B.A. Volkov, Solid State Commun. 50, 339 (1984)

48. N. A. Spaldin, M. Fiebig, M. Mostovoy, J. Phys.: Condens. Matter 20, 434203 (2008)

49. K.T. Delaney, M. Mostovoy, N.A. Spaldin, Phys. Rev. Lett. 102, 157203 (2009).

50. C. Ederer, N.A. Spaldin, Phys. Rev. B 76, 214404 (2007)

51. C. Ederer, Eur. Phys. J. B, DOI : $10.1140 /$ epjb/e2009-00274-4

52. B.B. Van Aken, J.-P. Rivera, H. Schmid, M. Fiebig, Nature 449, $702(2007)$

53. A.S. Zimmermann, B.B. Van Aken, H. Schmid, J.-P. Rivera, J. Li, D. Vaknin, M. Fiebig Eur. Phys. J. B, DOI : $10.1140 /$ epjb/e2009-00223-3

54. D.B. Litvin, Acta Cryst. A 64, 316 (2008)

55. D.B. Litvin, Eur. Phys. J. B, DOI : 10.1140/epjb/e2009-00229-9

56. K. Aizu, Phys. Rev. B 2, 754 (1970)

57. C.J. Fennie, Phys. Rev. Lett. 100, 167203 (2008)

58. I.E. Dzyaloshinskii, unpublished

59. F.W. Hehl, Y.N. Obukhov, J.-P. Rivera H. Schmid, Phys. Rev. A 77, 022106 (2008) 
60. F.W. Hehl, Y.N. Obukhov, J.-P. Rivera H. Schmid, Eur. Phys. J. B, DOI:10.1140/epjb/e2009-00203-7

61. D.L. Mills, I.E. Dzyaloshinskii, Phys. Rev. B 78, 184422 (2008)

62. A. Pyatakov, A. K. Zvezdin, Eur. Phys. J. B, DOI : 10.1140/epjb/e2009-00281-5

63. A. Pimenov, A.M. Shuvaev, A.A. Mukhin, A. Loidl, J. Phys.: Condens. Matter 20, 434209 (2008)

64. M. Schmidt, Ch. Kant, T. Rudolf, F. Mayr, A.A. Mukhin, A.B. Balbashov, J. Deisenhofer, A. Loidl, Eur. Phys. J. B, DOI : 10.1140/epjb/e2009-00215-3

65. R. Valdés Aguilar, M. Mostovoy, A.B. Sushkov, C.L. Zhang, Y.J. Choi, S-W. Cheong, H.D. Drew, Phys. Rev. Lett. 102, 047203 (2009)

66. A.B. Sushkov, M. Mostovoy, R. Valdes Aguilar, S.-W. Cheong, H.D. Drew, J. Phys.: Condens. Matter 20, 434210 (2008)

67. D. Senff, N. Aliouane, D.N. Argyriou, A. Hiess, L.P. Regnault, P. Link, K. Hradil, Y. Sidis, M. Braden, J. Phys.: Condens. Matter 20, 434212 (2008)

68. V. Zapf, F. Fabris, M. Kenzelmann, F. Balakirev, Y. Chen, C. Broholm, Magnetically-induced ferroelectric polarization in a molecule-based quantum magnet, abstract H12.00013, APS March Meeting (New Orleans, 2008)

69. J. Seidel, L.W. Martin, Q. He, Q. Zhan, Y.-H. Chu, A. Rother, M.E. Hawkridge, P. Maksymovych, P. Yu, M. Gajek, N. Balke, S.V. Kalinin, S. Gemming, F. Wang,
G. Catalan, J.F. Scott, N.A. Spaldin, J. Orenstein, R. Ramesh, Nat. Mater. 8, 229 (2009)

70. V.V. Shvartsman, S. Bedanta, P. Borisov, W. Kleemann, A. Tkach, P.M. Vilarinho, Phys. Rev. Lett. 101, 165704 (2008)

71. W. Kleemann, V.V. Shvartsman, S. Bedanta, P. Borisov, A. Tkach, P.M. Vilarinho, J. Phys.: Condens. Matter 20, $434216(2008)$

72. W. Kleemann, S. Bedanta, P. Borisov, V.V. Shvartsman, S. Miga, J. Dec, A. Tkach, P.M. Vilarinho, Eur. Phys. J. B, DOI : 10.1140/epjb/e2009-00222-4

73. A. Ohtomo, H.Y. Hwang, Nature 427, 423 (2004)

74. Satoshi Okamoto, A.J. Millis, Nature 428, 630 (2004)

75. S. Thiel, G. Hammerl, A. Schmehl, C.W. Schneider, J. Mannhart, Science 313, 1942 (2006)

76. A. Figotin, I. Vitebskiy, Phys. Rev. B 67, 165210 (2003)

77. N. Kida, T. Yamada, M. Konoto, Y. Okimoto, T. Arima, K. Koike, H. Akoh, Y. Tokura, Phys. Rev. Lett. 94, 077205 (2005)

78. D.V. Efremov, J. van den Brink, D.I. Khomskii, Nature Mater. 3, 853 (2004)

79. C. Ederer, N.A. Spaldin, Nature Mater. 3, 849 (2004)

80. H. Béa, M. Gajek, M. Bibes, A. Barthélémy, J. Phys.: Condens. Matter 20, 434221 (2008)

81. Y. Shimada, M. Matsubara, Y. Kaneko, J.P. He, Y. Tokura, Appl. Phys. Lett. 89, 101112 (2006) 